\title{
SINDACATO E CONSOLIDAMENTO DEMOCRATICO
}

\author{
di Davide Grassi
}

\section{Introduzione}

Le recenti transizioni democratiche in America latina e nell'Europa del Sud hanno messo in evidenza la speciale posizione dei sindacati tra le forze della società civile che reagiscono con un'accresciuta mobilitazione all'avvio della liberalizzazione in seno a regimi autoritari (Berins Collier e Mahoney 1997). Le organizzazioni sindacali, infatti, hanno generalmente la capacità di promuovere, in momenti critici, una mobilitazione più ampia ed efficace rispetto ad altri gruppi sociali. Esse non solo possiedono reti organizzative che, attraverso strutture più o meno permanenti, facilitano lo svolgimento di proteste e dimostrazioni, ma possono anche contare su schiere di militanti con specifici interessi in comune e su identità collettive politicamente definite. A differenza di gruppi come le organizzazioni degli studenti e le associazioni religiose o di quartiere, inoltre, $i$ sindacati possono colpire e danneggiare direttamente l'economia attraverso rivendicazioni salariali e scioperi (Valenzuela 1988, 3; Cella 1990, 17). La concomitanza delle transizioni politiche più recenti con una perdurante e diffusa crisi economica e con ripetuti tentativi di stabilizzazione e riforma hanno reso ancora più temibile questa capacità. Utilizzando poteri coercitivi garantiti dallo stato, o la semplice forza della persuasione, il sindacato, d'altra parte, può convincere la propria base ad aspettare sino a che le riforme producano dei risultati, contribuendo così a ridurre i livelli del conflitto sociale (Przeworski 1991, 181).

L'importanza politica delle organizzazioni dei lavoratori è dunque duplice e si fonda, da un lato, sulla loro capacità di promuovere e sostenere l'azione collettiva dei propri affiliati e, dall'altro, sul significato speciale che assume la capacità di conferire sostegno politico e di mobilitare l'opposizione (Berins 
Collier e Collier 1991, 41). Il primo aspetto è il risultato della presenza di lavoratori sindacalizzati in grandi centri di produzione, concentrati in spazi ristretti, o della loro collocazione strategica in punti critici del sistema produttivo e sociale, ciò che conferisce loro la capacità di sabotare il sistema economico e politico e rappresenta quindi un forte incentivo per una continua azione collettiva. Il secondo aspetto è più complesso e articolato: Payne (1965) ritiene, ad esempio, che in tutte le economie caratterizzate da un surplus di manodopera (è questo il caso dell'America Latina), i sindacati cerchino di rafforzare una situazione contrattuale naturalmente debole entrando nell'arena politica e sviluppando forme politiche di contrattazione. Nei sistemi politici relativamente centralizzati dell'America Latina, inoltre, $\mathrm{i}$ governi nazionali sono spesso coinvolti nelle fasi salienti dei più importanti conflitti sindacali ed i principali protagonisti sociali pensano abitualmente che il governo possa e debba fare qualcosa per la soluzione di tali conflitti. Data questa generale aspettativa, il mancato controllo della protesta può compromettere la stabilità del governo centrale.

L'importanza dell'azione collettiva del sindacato nella legittimazione dello stato, soprattutto nei sistemi politici a sviluppo più recente, è dunque cruciale (Waisman 1982). Il tema ricorrente è il seguente: gli elementi centrali della legittimazione, per come si è manifestata nei paesi europei, sono assenti o incompleti in altre aree geopolitiche e che i sindacati hanno svolto un ruolo centrale nel compensare questa mancanza. La fondamentale dipendenza dal sistema economico internazionale nel corso del ventesimo secolo, i cicli di denazionalizzazione economica che ne sono derivati ed il ruolo preminente di imprese straniere nello sviluppo economico, ad esempio, hanno reso la legittimazione del capitalismo e dello stato capitalista più problematica, in America Latina, rispetto a contesti nei quali lo sviluppo è stato in maggior misura controllato a livello nazionale (Hirschman 1979). O'Donnell $(1977 ; 1979 ; 1992)$ ricorda che nella regione la mediazione democratica tra società e stato è stata spesso sofferta, con una storia incerta di libere elezioni ed un rapporto problematico con le principali libertà civili. Altre forme di mediazione, come il nazionalismo ed il populismo, hanno svolto un ruolo più importante. Il settore popolare è stato portatore della mediazione populista ed un importante veicolo di quella nazionalista: le sue più rilevanti forme organizzative sono stati i sindacati urbani ed, in alcuni casi, quelli contadini. Attra- 
verso la cooperazione con il sindacato, dunque, lo stato può affrontare ed iniziare a risolvere importanti problemi di legittimità ${ }^{1}$. Oppure, il movimento dei lavoratori può costituire uno strumento di protesta contro le politiche dello stato ed indebolirne la legittimità. La prospettiva incentrata su nazionalismo, populismo e legittimità, per concludere, chiarisce ulteriormente perché l'azione collettiva del sindacato possa, perlomeno in certe regioni del globo ed in certi momenti storici, divenire una potente forza politica e perché le risposte dello stato alle proteste dei lavoratori siano, allo stesso modo, egualmente importanti (Berins Collier e Collier 1991, 44)².

\section{Consolidamento democratico e relazioni industriali}

Queste osservazioni suggeriscono che l'importanza ed il ruolo delle organizzazioni sindacali nei processi di democratizzazione, in America Latina ed Europa del Sud, interessano anche le successive fasi di consolidamento dei nuovi regimi. Una loro analisi presuppone, dunque, un chiarimento del concetto di consolidamento democratico e delle dimensioni che lo caratterizzano. Le interpretazioni offerte in questo senso sono state

1 Uno strumento recentemente utilizzato per raggiungere obiettivi di questo tipo è quello della concertazione economico-sociale. Contro una concezione puramente conflittuale della politica, i propugnatori del patto sociale immaginano situazioni nelle quali tutti possano beneficiare o, quantomeno, tutti siano posti nella condizione di soffrire allo stesso modo. La concertazione implica, quindi, non solo che i diversi interessi siano resi compatibili, ma anche, e soprattutto, una dimensione intersoggettiva di creazione collettiva e di legittimità (Dos Santos 1985). Attraverso la concertazione si intendono anche armonizzare i meccanismi di regolazione delle relazioni economico-sociali e fondamentalmente il conflitto capitale-lavoro, attraverso la partecipazione istituzionale e la corresponsabilità dello stato e dei settori sociali organizzati nel disegno e nell'esecuzione di politiche socio-economiche che permettano di governare economie in crisi (Thompson 1995, 21).

2 Oltre al volume di Berins Collier e Collier, di cui si discute ampiamente, le più importanti analisi dei movimenti sindacali in America Latina sono state quelle di Spalding (1977), Bergquist (1986) e Epstein (1989). Il primo è uno studio dei lavoratori urbani organizzati, dalle origini agli anni settanta, formulato nel linguaggio delle teorie della dependencia, e presta particolare attenzione al ruolo delle fluttuazioni economiche internazionali e degli interessi politici della borghesia nelle nazioni più sviluppate. Per Bergquist, invece, è la struttura delle esportazioni che determina sia il potenziale di crescita economica di ogni paese, che quello organizzativo dei sindacati e della sinistra in generale. Epstein, per concludere, analizza il ruolo dell'autonomia o subordinazione sindacale allo stato nel contesto dei recenti processi di democratizzazione politica. Il rapporto tra democratizzazione e sindacati è anche affrontato, in un ottimo lavoro in lingua italiana, da Cuevas (1986). 
diverse ed articolate: secondo alcuni, un regime si consolida quando le elezioni politiche e le libertà ad esse collegate si istituzionalizzano e non sono fatti valere poteri di veto nei confronti di candidati democraticamente eletti da parte di militari o superstiti enclavi autoritarie (Linz 1990). Nessuno dei maggiori gruppi politici e delle più importanti istituzioni pensa che sia praticabile un'alternativa al processo democratico come mezzo per accedere al potere: in una parola la democrazia viene percepita come the only game in town. A questa definizione «minimalista» del consolidamento, fondata sul ruolo centrale delle elezioni, è stata contrapposta una concezione più estesa, incentrata sul raggiungimento di un alto grado di istituzionalizzazione delle organizzazioni democratiche complesse, come l'esecutivo, i partiti, il potere legislativo e quello giudiziario (Gunther, Diamandouros e Puhle 1995). Secondo questa interpretazione il consolidamento sarebbe il processo di congelamento, nei suoi caratteri essenziali e di adattamento, in quelli secondari, delle strutture e norme democratiche, indotto dal trascorrere del tempo (Morlino 1986).

Il compromesso democratico garantisce l'appoggio o la neutralità di attori chiave nel processo di radicamento della democrazia: militari, imprenditori pubblici e privati e lavoratori organizzati. Per il buon esito del consolidamento è spesso sufficiente la neutralità, o la neutralizzazione, dei primi, mentre è importante l'appoggio attivo dei gruppi che operano negli ambiti produttivi. Le élites civili devono, quindi, innescare una strategia che induca i militari ad accettare il nuovo assetto politico e a restare definitivamente nelle caserme. Esse devono, inoltre, garantire gli interessi degli imprenditori privati, sia direttamente attraverso i consueti organi esecutivi e legislativi, sia indirettamente attraverso i partiti. La cooperazione dei sindacati, infine, è cruciale per il potenziale di conflitto sociale di cui sono capaci: spesso le vicende ed i problemi del movimento sindacale identificano potenzialità e limiti dei nuovi regimi liberali, la loro capacità di evolversi verso assetti liberaldemocratici ed i margini di competizione fra l'organizzazione pluralistica della società, centrata sulla rappresentanza funzionale degli interessi, e le regole della democrazia politica (Cella 1995).

Gli aspetti del consolidamento che intendo sottolineare in questo articolo riguardano appunto il campo dell'azione collettiva del sindacato e delle relazioni industriali e si inquadrano, quindi, in una concezione ampia del concetto. Il conflitto tra le 
domande dei principali agenti produttivi, lavoratori organizzati ed imprenditori, costituisce il nucleo attorno a cui sono organizzate le moderne democrazie capitaliste: col tempo esso si è materializzato in una serie di istituzioni che permettono una loro soluzione pacifica. $\grave{E}$ evidente, dunque, la sua importanza per valutare il grado di complessivo consolidamento democratico raggiunto dal sistema politico.

In questo ambito un primo momento è quello del riconoscimento delle parti nel rapporto sociale e della creazione di norme e procedure attraverso le quali queste giungono a contrattare collettivamente il salario e le condizioni di lavoro. Il governo regola, innanzitutto, l'esistenza dei sindacati e la loro capacità d'azione: esso può, ad esempio, porre condizioni per il loro riconoscimento e decidere che la rappresentanza dei lavoratori sia monopolio esclusivo di una sola organizzazione, o di quella più rappresentativa, non permettendo, quindi, o rendendo particolarmente difficile, il sorgere di strutture sindacali alternative. $\mathrm{O}$, ancora, può riservarsi ampi poteri di controllo sulle attività svolte e, in alcuni casi, la facoltà di sciogliere l'organizzazione. L'esecutivo può, infine, disciplinare il ricorso da parte del sindacato a forme di conflitto industriale, e soprattutto allo sciopero, regolandone le procedure, limitandone il diritto e restringendone l'applicazione, oppure cercando di imporre soluzioni obbligatorie alle vertenze (Bronstein 1995, 196-197; Ermida Uriarte 1993, 34). Particolarmente importante è anche l'istituzione di rapporti autonomi e ben definiti tra lavoratori organizzati ed imprenditori, che sostituisca la regolamentazione imposta dallo stato sui principali aspetti economici ed organizzativi del rapporto di produzione. In alcuni casi, infatti, è il governo che regola direttamente, di volta in volta, aspetti cruciali del rapporto e specialmente la sua componente salariale. Oppure può specificare le modalità con cui ha luogo la contrattazione industriale, restringendola alle sole forme decentralizzate, a livello delle singole aziende: le decisioni sono messe in pratica per decreto ed imposte a lavoratori ed imprenditori. Il requisito dell'omologazione degli accordi sottoscritti dalle parti, inoltre, se esteso ad un controllo della sostanza delle decisioni, riduce la loro autonomia ed elimina l'accettazione spontanea della procedura e dei suoi risultati. I gruppi d'interesse vengono esclusi dalla procedura e non esistono istituzioni specifiche per l'espressione delle rispettive posizioni e per una loro eventuale conciliazione. Laddove invece venga riconosciuto il diritto delle 
parti di decidere autonomamente il contenuto e le forme del loro rapporto, questo può essere più o meno protetto, enunciato in semplici leggi ordinarie o sancito in modo più duraturo e solenne nella stessa Carta costituzionale, oppure può essere definito solo nelle sue forme più generali o specificato e reso operativo in leggi e regolamenti di attuazione.

Un'analisi dell'impatto dell'azione sindacale sui processi di consolidamento richiede anche un esame approfondito dei rapporti tra sindacato e partiti politici e della partecipazione di questi alle coalizioni che sostengono e consolidano il passaggio a forme democratiche di governo. $\grave{E}$ attraverso il filtro della partecipazione dei partiti al governo, infatti, ed in conseguenza della pressione che il sindacato esercita su di essi, che si manifesta pienamente la sua capacità dirompente sugli equilibri politici dei nuovi regimi. L'esistenza di un rapporto di cooperazione e reciproco sostegno tra partiti e sindacati è rivelato non solo dalla comune ideologia e dallo sviluppo storico e di legami organizzativi. Esso è confermato anche dall'esistenza di programmi più o meno comuni, dalla capacità o inadeguatezza del sindacato nell'elaborare strategie economiche e sociali indipendenti, dalle modalità di selezione, autonoma o imposta, dei rispettivi dirigenti e quadri direttivi e, ancora, dell'erogazione di finanziamenti pubblici.

\section{Alcune ipotesi esplicative}

Le interpretazioni dei processi di consolidamento delle relazioni industriali e delle posizioni sindacali nell'ambito dei nuovi regimi democratici non sono state molto numerose. Un primo filone si è ricollegato a momenti storici cruciali. In America Latina, dopo la crisi mondiale degli anni trenta, si impone una fase di industrializzazione per sostituzione delle importazioni, nella quale assumono nuova importanza i mercati interni e le masse nazionali che li alimentano e li sostengono. In questa fase l'incorporazione delle masse è attuata attraverso la partecipazione decisiva dello stato e prende forma in una legislazione e schemi pensionistici favorevoli ai lavoratori: si riconoscono $\mathrm{i}$ primi sindacati e vengono promulgati i primi codici del lavoro. In cambio i governi cercano di imporre forme di controllo sulle attività sindacali, cooptandone i dirigenti e mirando ad una generale moderazione delle domande. Successivamente si afferma 
un capitalismo dipendente e transnazionale, in cui l'apertura all'esterno corrisponde a un trasferimento di capitali dalla periferia al centro economico e a un controllo straniero dell'economia, concentrato in alcuni settori. Ė questa una fase di esclusione dei sindacati. A partire dagli anni sessanta, essi subiscono una repressione crescente, che culmina, con la proclamazione dei regimi autoritari, nella loro repressione e dissoluzione $(\mathrm{Za}$ pata 1993). Un argomento simile è sviluppato in Berins Collier e Collier (1991). La fase cruciale per comprendere la natura del ruolo dei sindacati nelle società latinoamericane contemporanee, e le caratteristiche dei regimi politici in cui operano, sarebbe la fase della loro incorporazione, durante il periodo economico dell'industrializzazione per sostituzione delle importazioni. L'implicazione più interessante della teoria è che i modi dell'incorporazione lascerebbero un segno istituzionale duraturo. L'incorporazione attuata dallo stato provoca una radicalizzazione del movimento sindacale, mentre quella attraverso i partiti, dopo una reazione violenta che può assumere le forme del colpo di stato, presenterebbe un decorso più favorevole al rafforzamento democratico. Quest'ultimo sarebbe favorito dalla presenza nel sistema politico di una stabile maggioranza di centro, con la quale il sindacato sia organizzativamente affiliato (Berins Collier e Collier 1991, 765-768).

Un secondo filone interpretativo pone l'accento su alcune caratteristiche organizzative. Il consolidamento delle relazioni industriali e della democrazia sarebbero, in questa prospettiva, funzione di un sindacalismo forte, politicamente significativo e ideologicamente riformista, di governi progressisti che esercitano una capacità egemonica sul sindacalismo e di forme corporative di intermediazioni degli interessi, caratterizzate da un alto grado di concentrazione e centralizzazione. In tali condizioni alcuni autori aggiungono anche la presenza di un capitalismo sviluppato, in grado di ricompensare materialmente i lavoratori, e di organizzazioni sindacali unificate e centralizzate, capaci di determinare e porre in essere obiettivi e strategie (Tironi 1988). In sintesi, sarebbero quindi, necessarie forti confederazioni sindacali e la presenza al governo di un partito affiliato al sindacato, capace di usare le risorse pubbliche per promuovere e garantire un patto sociale, oltre ad economie sufficientemente stabili e forti (Blake 1994, 389). A questo risultato contribuiscono anche una certa apertura politica nei confronti dei sindacati durante il periodo autoritario, una sostanziale moderazione dei nuovi lea- 
der sindacali, interessati a preservare il processo di transizione democratica e, secondo alcuni, una posizione politica decentrata del partito affiliato al sindacato, che è parte della coalizione che amministra la transizione, ma non partecipa al governo con incarichi diretti (Valenzuela 1988) . $^{3}$.

Un terzo gruppo di spiegazioni fa invece leva su fattori preminentemente economici. Secondo queste interpretazioni il rafforzamento di alcune delle caratteristiche del sistema di relazioni industriali è una funzione delle condizioni economiche prevalenti al momento della transizione. Crisi economiche di lieve entità - diminuendo la distanza tra ciò che i lavoratori possono conseguire esercitando forme di moderazione salariale e ciò che riuscirebbero a conquistare affidandosi alla sola forza contrattuale - renderebbero più probabili le intese tra sindacati, imprenditori e governo e, quindi, accordi economici tali da facilitare la transizione, senza peraltro condurre necessariamente ad una istituzionalizzazione completa delle procedure per la soluzione dei conflitti e delle rivendicazioni industriali (Lange 1984). È questa la tesi generalmente sostenuta, ad esempio con riferimento al caso spagnolo (Pérez-Díaz 1986). Per altri, invece, durante le crisi economiche i patti sarebbero più difficili, poiché gli imprenditori potrebbero comunque ottenere condizioni più vantaggiose dai lavoratori contrattando a livello di impresa (Blake 1994) ${ }^{4}$. Pur riconoscendo l'importanza centrale di alcuni processi economici nelle fasi dello sviluppo politico, si è sottolineato che, in ogni caso, il loro rapporto è spesso meno diretto di quanto suggerito da parte della letteratura. In particolare, l'arena politica non risponde in modo fluido e costante ai cambiamenti socioeconomici, ma può ostacolarli, rispondendo, per periodi di tempo relativamente lunghi, ad una sua logica politica autonoma ed agli interessi costituiti dei politici (Berins Collier e Collier 1991).

3 Si è sovente sottolineata l'importanza relativa dei sindacati in contesti economici e sociali, come quelli dell'America Latina, nei quali prevalgono rapporti di tipo informale (Sofer 1980). In questo senso, la presente ricerca deve essere intesa come un'analisi di aspetti importanti di un settore economico particolare, quello formale appunto, che seppure minoritario in senso strettamente numerico, ha svolto un'importante funzione politica, dovuta alla sua particolare capacità di mobilitazione collettiva (Portes e Walton 1975).

4 Per una vasta sintesi della letteratura sul rapporto tra sviluppo economico e fenomeni politici in America Latina, con particolare riferimento al nesso tra fase di sostituzione delle importazioni e periodo di incorporazione delle masse popolari e populismo, vedi Collier (1979). 
Sindacato, consolidamento delle relazioni industriali e consolidamento della democrazia

Sono già state esaminate le principali ragioni dell'importanza dei sindacati nel contesto delle transizioni e del consolidamento democratico. L'importanza economica e politica del movimento dei lavoratori spiega perché siano cruciali le scelte di controllo e di mobilitazione del sostegno sindacale. Dal punto di vista dei partiti al governo si pone il problema della disciplina di questa forza potenzialmente dirompente, ma si presenta anche l'opportunità di mobilitare nuove basi di appoggio politi$\mathrm{co}^{5}$. Da un punto di vista sindacale, la partecipazione, come mezzo per difendere gli interessi dei lavoratori, comporta il rischio di cooptazione e perdita della propria autonomia organizzativa, ma anche il potenziale vantaggio di conseguire proficui accordi di scambio politico con i partiti al potere.

Alla luce di queste osservazioni sono due le dimensioni che possono essere utilmente utilizzate nella costruzione di un modello esplicativo dei processi di consolidamento delle relazioni industriali e del nuovo regime democratico. La prima è l'incorporazione politica del sindacato, cioè l'insieme dei rapporti storici, organizzativi ed ideologici, che il partito al potere intrattiene con il movimento sindacale e che permettono l'instaurazione o il mantenimento di canali preferenziali di comunicazione e cooperazione nell'attuazione delle politiche di governo. Questo fattore è strettamente connesso alla mobilitazione o smobilitazione del sindacato, cioè al processo con cui il governo trasferisce o sottrae al controllo del sindacato una serie di risorse, di tipo economico e legale. Il governo, ad esempio, può prestare aiuti in denaro, restituire le proprietà appartenute ai sindacati operanti durante i regimi autoritari, affidare loro la gestione di enti previdenziali, richiedere per legge il pagamento dei contri-

5 In America Latina e in Europa del Sud, quando il regime autoritario non si è dimostrato capace di risolvere importanti problemi sociali ed economici, i moderati della coalizione al potere hanno spesso conquistato nuovi spazi e preferito liberalizzare e persino democratizzare il sistema, piuttosto che lottare, in modo inefficace, contro un'opposizione sempre più mobilitata e combattiva. Quando prevale una situazione di crisi irrisolta, dunque, i riformatori tendono a promuovere forme di incorporazione sindacale, come risorsa politica nel conflitto con le componenti più conservatrici della coalizione al potere. La mobilitazione è stata perseguita durante le fasi della democratizzazione in misura inversamente proporzionale alla forza politica dei «falchi» (Berins Collier e Collier 1991, 748). 
buti sindacali, regolare in maniera favorevole la disciplina dell'occupazione $\mathrm{e}$, in generale, facilitare le attività sindacali dentro e fuori il posto di lavoro. Oppure può negare gli aiuti, limitare la partecipazione del sindacato ad attività di assistenza, imporre oneri fiscali ed organizzativi e, in generale, disciplinare in modo limitativo i poteri e le attività dell'organizzazione. Se la transizione democratica e il successivo consolidamento sono spesso associati ad un rafforzamento dei sindacati e dei movimenti operai, la collaborazione con partiti politici amici ha permesso di contenere in modo più efficace congiunture macroeconomiche spesso avverse (Valenzuela 1994, 148-149). La vicinanza tra sindacato e governo assicura un maggior controllo della militanza dei lavoratori, con una riduzione degli scioperi e delle manifestazioni di protesta ed un contenimento delle domande salariali. Il beneficio sulle prospettive di consolidamento è, dunque, duplice: da un lato diminuisce la pressione salariale, causa di inflazione e di innumerevoli tensioni economiche, dall'altro migliora l'ordine pubblico e si irrobustisce lo schema della rappresentanza e mediazione politica attuata attraverso il sistema dei partiti (Przeworski 1991)

La seconda dimensione ha a che vedere con la maggiore o minore autonomia delle relazioni tra sindacati e datori di lavoro. Si tratta cioè di vedere se questi rapporti siano definiti direttamente dallo stato, che interviene risolvendo particolari conflitti, di natura salariale o di organizzazione del lavoro, oppure se essi siano lasciati alla libera determinazione dei gruppi e alla loro interazione. Ciò non significa che lo stato e il governo non possano cercare di convincere le organizzazioni operaie e degli imprenditori a conformarsi a certe regole di condotta per risolvere i loro problemi più gravi. Significa, piuttosto, che dovranno sforzarsi di discutere con degli attori indipendenti anziché imporre il proprio punto di vista a delle organizzazioni sottoposte: la differenza tra i due modi d'azione è fondamentale (Valenzuela 1988) ${ }^{7}$.

6 In questo scambio il sindacato si rafforzerebbe organizzativamente e finanziariamente, anche a scapito di sindacati rivali ed otterrebbe forme indirette, ma molto importanti, di assistenza ai propri affiliati, quali spese pubbliche per salute, educazione, previdenza ed assistenza sociale.

7 Il carattere sostanzialmente eteronomo delle relazioni industriali in America Latina ha molteplici cause. Innazitutto una causa economica. La struttura industriale del continente latinoamericano è stata a lungo caratterizzata da enclavi produttive, che hanno favorito uno sviluppo sindacale limitato e frammentato. Per costituire dei sistemi ge- 
Il governo può anche intervenire nominando i dirigenti dell'organizzazione sindacale o fissando i metodi per la loro elezione e definendo le strategie adottate dalle diverse organizzazioni. Nei regimi latinoamericani e dell'Europa del Sud, la supremazia dello stato nelle relazioni industriali ha spesso avuto per obiettivo il controllo delle rivendicazioni salariali e della militanza politica dei lavoratori (Bronstein 1995; Ermida Uriarte 1993; Touraine 1988). La partecipazione preponderante dello stato nella gestione delle relazioni industriali impedisce il rafforzamento di un sistema fondato sulla espressione diretta dei principali attori sociali e sulla loro capacità di formulare i rispettivi punti di vista e giungere a dei compromessi accettabili ${ }^{8}$. I conflitti sociali, al contrario, hanno un esito più favorevole quando le organizzazioni operaie e degli imprenditori possono risolvere le loro controversie riducendo al minimo l'intervento diretto dello stato (Valenzuela 1994, 144-148) ${ }^{9}$. Privati di una loro efficace rappresentanza, i lavoratori sottraggono legittimità al sistema delle relazioni industriali ed al nuovo regime democratico, evitando una partecipazione attiva nel nuovo regime e, in casi estremi, appoggiando soluzioni eversive ed autoritarie. Gli effetti sul consolidamento democratico sono stati, dunque, negativi: l'effetto favorevole di un maggiore controllo salariale e sociale è

nerali di disciplina delle relazioni del lavoro, dunque, non si è potuto contare sulla contrattazione autonoma delle parti: si è, al contrario, dovuti ricorrere a formulazioni generali della legge, dispensate dalle autorità centrali. Da un punto di vista storico-culturale ha operato la derivazione del diritto latinoamericano dal diritto romano, un sistema giuridico scritto e centrato sull'autorità dello stato, che identifica il diritto con la legge e che ha sancito questo binomio con l'adozione di codici ispirati alla tradizione francese. C'è poi un fattore politico, l'intento, da parte dello stato latino americano, di controllare politicamente l'azione del sindacato. Questa ossessione è evidenziata dal carattere chiaramente asimmetrico della regolamentazione dei sindacati e delle associazioni di datori di lavoro, molto più blanda, e la poca propensione alla protezione, oltre che alla regolamentazione, dell'azione sindacale. Per ultimo si può ricordare la stessa debolezza dei movimenti sindacali, che non permette una resistenza efficace alle imposizioni governative e che, anzi, trova in esse una serie di garanzie minime che difficilmente potrebbe conseguire in altro modo.

8 La partecipazione dello stato a tutela del rapporto di lavoro individuale, al contrario, svolge un ruolo comunque positivo.

9 E utile ricordare che le due dimensioni qui impiegate sono analiticamente distinte. Nella mobilitazione o smobilitazione sindacale il governo fornisce o sottrae risorse economiche e legali al sindacato, che può rimanere, però, controllato da una dirigenza autonoma ed indipendente, espressione della base sindacale o degli stessi quadri dell'organizzazione. L'autonomia o subordinazione del sindacato e del sistema di relazioni industriali si riferisce appunto alla maggiore o minore capacità delle parti sociali di darsi, di propria scelta, le regole della reciproca interazione e dello svolgersi delle attività interne dell'organizzazione e di realizzare queste attività in accordo con tali regole. 
stato vanificato dall'intervento governativo palesemente volto alla protezione degli interessi di una sola parte, che, spesso, è sfociato in aspri conflitti sociali e nella repressione più cruenta (Buchanan 1991) ${ }^{10}$.

Il doppio requisito, della mobilitazione o smobilitazione politica del sindacato e dell'autonomia o dipendenza del sistema delle relazioni industriali nei confronti del governo, facilita una prima classificazione e fornisce una interpretazione iniziale dei casi e delle modalità di incorporazione dei sindacati nelle fasi di transizione a nuovi regimi democratici e durante il successivo consolidamento. La tabella 1 illustra le possibili combinazioni. Quando il sindacato è politicamente smobilitato e soggetto ad un controllo rigoroso e discrezionale da parte dello stato, un caso che si verifica ad esempio in Bolivia, la militanza sindacale viene contenuta con leggi e regolamenti che subordinano al beneplacito delle autorità centrali ogni iniziativa e rivendicazione. L'azione dello stato giunge sino alla repressione più aperta ed $\mathrm{i}$ sindacati vengono permanentemente esclusi da ogni processo di formulazione ed attuazione dei programmi socio-economici ${ }^{11}$. Se il sindacato è smobilitato, ma lo stato non esercita un controllo significativo sulle relazioni industriali, come in Uruguay, la situazione sarà caratterizzata da un forte conflitto sociale, ma questo, svolgendosi direttamente tra lavoratori organizzati e datori di lavoro, non ridurrà il consenso sulla legittimità della democrazia in quanto tale. La contrattazione e la sfera salariale verranno definite dalla forza rispettiva delle organizzazioni sociali e saranno de-

10 Nell'Europa del Sud lo sviluppo economico durante gli anni settanta è certamente più omogeneo e avanzato e maggiore è l'attenzione del potere politico nei confronti di un sindacato che, soprattutto negli anni che seguono la transizione, è relativamente forte e capace di mobilitare enormi masse di lavoratori. In Spagna, dunque, il sistema delle relazioni industriali acquisisce un carattere più aperto ed autonomo, nonostante permanga un intento conciliatore e mediatore da parte delle autorità pubbliche, che favoriscono e cercano di strutturare la contrattazione e la concertazione tra le parti sociali.

11 Nonostante la prevalente retorica neoliberale questa rimane la natura del rapporto tra stato e sindacato in Bolivia: ogni qualvolta le rivendicazioni sindacali appaiono eccessive e mettono in pericolo, a giudizio dell'esecutivo, le misure di austerità e l'equilibrio economico, il governo è pronto a dichiarare lo stato d'assedio e a proibire gli scioperi. D'altro lato, in seguito alla crisi economica, le relazioni industriali boliviane sono state alleggerite e le relazioni con gli imprenditori sono state affidate, nel quadro di una generale perdita di forza delle organizzazioni sindacali, alla capacità contrattuale delle parti e allo strumento degli accordi a base locale. Non di diminuzione del ruolo dello stato si deve parlare, dunque, ma semplicemente di nuove, meno costose strategie per il controllo dei sindacati da parte dello stato. 
terminate anche dalla congiuntura economica nazionale ed esterna. In un contesto economico, come quello latinoamericano, spesso segnato da gravi crisi e caratterizzato da una forza sindacale di livello medio, il risultato più comune sarà quello di elevati livelli di conflitto industriale, scioperi e dimostrazioni. In questo caso, tuttavia, lo stato si limiterà a controllare dall'esterno l'azione sindacale, per evitare che degeneri in disordini sociali incontrollabili, ed inviterà occasionalmente l'organizzazione ad esporre le proprie posizioni nel tentativo di giungere a soluzioni concertate, senza però concludere accordi formali e piegandosi solo raramente a concessioni economiche.

Nel caso in cui il governo mobilita il sindacato, ma sceglie di non intromettersi nella gestione delle sue strategie e delle relazioni con gli imprenditori, come in Spagna, i diritti sindacali godono di un'ampia tutela, la contrattazione è libera e tende ad essere centralizzata per rami di produzione, ma senza l'imposizione di regole formali stabilite dal governo. Il contenuto della contrattazione collettiva è liberamente determinato dalle parti e il diritto di sciopero è riconosciuto e in alcuni casi limitato. Patti socioeconomici tra imprenditori, sindacati e governo vengono conclusi, ma i costi che comportano finiscono spesso col convincere una parte della base sindacale che $\mathrm{i}$ sacrifici richiesti sono eccessivi e che è preferibile perseguire interessi più immediati. Quando, infine, lo stato controlla le relazioni industriali e si impegna in una capillare mobilitazione sindacale, i diritti dei lavoratori, di libera contrattazione, sciopero, ed autonomia dai partiti, vengono solennemente affermati e le forme della contrattazione salariale tendono a rivestire una forma centralizzata, mentre i sindacati partecipano spesso alla formulazione ed all'attuazione di piani economici e sociali di interesse nazionale. Questi principi, tuttavia, possono essere accompagnati (è proprio questo il caso del Venezuela), da forme di controllo della dirigenza sindacale e delle attività dell'organizzazione, che spesso sono solo formalmente autonome mentre, in altri casi, come per gli scioperi, si impone una rigida disciplina ${ }^{12}$.

$12 \mathrm{Da}$ un altro punto di vista questo schema permette un'interpretazione della conclusione di accordi e patti tra stato, sindacati e partiti. L'esito della concertazione sociale tra governo e sindacati è multiforme: il sindacato può innanzitutto sottoscrivere un patto esplicito con le altre parti sociali, che comporta obblighi di contenimento delle proprie domande e rivendicazioni (Spagna e Venezuela). Oppure tale partecipazione è più limitata e ridotta a contatti, anche intensi, che non approdano ad un patto vero e proprio, ma si risolvono in una serie di incontri di informazione o tentativi di predi- 
TAB. 1. Forme di consolidamento del sistema delle relazioni industriali (e forme del consolidamento democratico)

Autonomia delle relazioni industriali

Ampia Limitata

Sì Pluralismo industriale (consolidamento stabile e legittimo)

SPAGNA

Inclusione controllata

(consolidamento senza

legittimità)

VENEZUELA

Mobilitazione

politica del

sindacato

\begin{tabular}{llc} 
No & $\begin{array}{l}\text { Esclusione conflittuale } \\
\text { (consolidamento } \\
\text { socialmente instabile) } \\
\text { URUGUAY }\end{array}$ & $\begin{array}{l}\text { Esclusione e repressione } \\
\text { (consolidamento instabile } \\
\text { e illegittimo o crisi) } \\
\text { BOLIVIA }\end{array}$ \\
\hline
\end{tabular}

I quattro casi esaminati esibiscono diversi gradi di consolidamento delle relazioni industriali. Più forte laddove alla transizione segue una mobilitazione delle organizzazioni sindacali, diretta da partiti politici amici, più debole laddove questa non si concretizza, oppure il governo contrasta apertamente l'azione dei sindacati ${ }^{13}$. In Venezuela, tuttavia, l'assenza di una autentica

sporre strategie in comune che però non riescono a concretizzarsi. Se un patto, poi, viene concluso, in genere non viene messo in pratica (Uruguay). In altri casi, infine, il sindacato è emarginato e viene semplicemente tollerato o addirittura attivamente represso (Bolivia, negli ultimi anni). Il caso del patto socioeconomico è particolarmente interessante: il fine immediato di questi patti è quello di riattivare la competitività economica $\mathrm{e}$ la stabilità dei prezzi e dell'occupazione, attraverso un sistema centralizzato di regolamento dei salari, basato sul requisito della moderazione sindacale: essi forniscono, però, anche $\mathrm{i}$ canali attraverso i quali alcuni importanti conflitti economici e politici vengono istituzionalizzati e rappresentano, perciò, un terreno ideale di analisi del rapporto tra $\mathrm{i}$ principali attori sociali nelle fasi della transizione. Come si evince dalla tabella 1 , la loro conclusione è particolarmente favorita da una precedente mobilitazione politica del sindacato, ma la loro efficacia dipende dall'assenza di controlli eccessivi sul sindacato e sulle relazioni industriali in genere (Ilo 1996; Przeworski 1988; Dos Santos 1985; Cordova 1985; Lechner 1985).

$13 \mathrm{Nel}$ caso in cui all'azione ostile del governo corrisponda un sindacato organizzativamente e politicamente debole questi potrà astenersi da forme dirette di intervento giuridico o repressivo e lasciare che la diseguale distribuzione delle forze rispetto agli imprenditori, o politiche macroeconomiche mirate ad indebolire indirettamente la capacità contrattuale dell'organizzazione, determinino una sostanziale moderazione delle domande ed un contenimento dei livelli di conflitto industriale. In questo caso l'intervento solo indiretto del governo, che mantiene formalmente l'autonomia sindacale, evita il sorgere di problemi di legittimità e riduce il potenziale dirompente delle rivendicazioni 
autonomia nel sistema delle relazioni industriali produce una seria crisi di legittimità, mentre in Spagna le istituzioni create durante la transizione sopravvivono e si radicano senza la continua imposizione ed il costante controllo dello stato. Il consolidamento del sistema di relazioni industriali è sicuramente più compiuto nel secondo caso. In Uruguay gli effetti benefici di una maggiore autonomia dallo stato consentono un maggiore rafforzamento dei rapporti tra sindacati ed imprenditori, che rimangono tuttavia caratterizzati da una elevata conflittualità. In Bolivia, invece, la persistente presenza di uno stato controllore e repressore inasprisce gli effetti negativi sul consolidamento. La serie di studi del caso che segue scandisce in modo specifico il rapporto tra rafforzamento delle relazioni industriali e consolidamento della democrazia.

I casi

La Spagna rappresenta un caso di consolidamento democratico portato a buon fine, nel quale le principali istituzioni politiche ed il sistema di relazioni industriali hanno acquisito un grado soddisfacente di legittimità complessiva. Solo una volta, nel 1981, ha avuto luogo un tentativo di colpo di stato, che è stato rapidamente debellato. L'opposizione armata al governo democratico è stata promossa specialmente da movimenti separatisti, la cui attività precede la creazione della democrazia ed è diminuita d'intensità ${ }^{14}$. Nel 1977 i partiti politici rappresentati in parlamento, inclusa l'opposizione, firmano un accordo politico, i Patti della Moncloa, che li obbliga al rispetto dei principi democratici e di alcune politiche economiche concordate con il governo. La maggior parte dei partiti è favorevole ai sindacati più rappresentativi: la Unión del centro democrático (Ucd), una coalizione di gruppi centristi, e il Partido socialista obrero español (Psoe) facilitano un modello di concertazione socioeconomica moderato, caratterizzato dalla conclusione di ampi patti

sindacali, contribuendo ad un consolidamento complessivo del nuovo regime, i cui contenuti democratici rimarranno, tuttavia, limitati.

14 Sulla transizione e consolidamento della democrazia in Spagna si vedano PérezDíaz (1993); Tezanos, Cortarelo e de Blas (1989); Foweraker (1987); Maravall (1982). 
nazionali sottoscritti da sindacati, governo ed imprenditori ${ }^{15}$. Il Partito comunista non viene escluso dalla partecipazione alla competizione elettorale, nonostante le pressioni in questo senso siano molto forti, soprattutto da parte dell'establishment militare. La sua accettazione è però subordinata all'accoglimento di alcune condizioni, istituzionali e di programma, dettate dal nuovo governo. Tra l'altro, i comunisti si impegnano a sostenere una politica di moderazione dei salari e di contenimento delle domande sociali, che ne limita decisamente l'immagine di partito d'opposizione e di possibile alternativa politica.

I diritti dei lavoratori sono tutelati direttamente dalla Costituzione del 1978, che trova una prima attuazione nell'Estatuto de los Trabajadores, approvato nel 1980. Il governo non ha poteri speciali riguardo al riconoscimento dei sindacati, che si possono formare liberamente, e non interviene nei processi di formazione dei contratti con procedimenti di omologazione degli accordi collettivi; restringe però in maniera significativa il diritto di sciopero (McElrath 1989, 164). Decisivo, comunque, è che le parti abbiano potuto, dopo il 1979, trattare autonomamente accordi salariali senza l'intervento coercitivo del governo. La soluzione obbligatoria dei conflitti industriali, infine, imposta in un primo momento dal governo, è stata successivamente limitata dal potere giudiziario (McElrath 1989, 153). Il diritto alla contrattazione collettiva è soggetto ad alcuni restrizioni il cui obiettivo è principalmente di razionalizzare e rendere più efficiente la contrattazione tra le parti anziché di introdurre controlli preventivi e censure: così, ad esempio, solo i sindacati che si assicurano almeno il dieci per cento dei rappresentanti di fabbrica sono ammessi a trattare a livello nazionale. I contratti collettivi, inoltre, vengono resi obbligatori per tutti i lavoratori

15 Allo stesso tempo i partiti, durante i periodi di rispettivo controllo del governo, cercano di rafforzare i sindacati a loro più vicini ed indebolire quelli meno docili. Il governo Suárez si oppone, ad esempio, al tentativo del sindacato comunista Comisiones obreras, allora maggioritario, di ottenere il riconoscimento legale del vecchio sindacato franchista, che aveva infiltrato durante la dittatura. Il riconoscimento avrebbe comportato la creazione di un'organizzazione sindacale unitaria e radicalizzata. Il primo ministro, invece, favorisce un maggiore pluralismo sindacale, che dà la possibilità al sindacato socialista, allora minoritario e più moderato, di partecipare alle elezioni sindacali in condizioni più favorevoli. Il governo stabilisce anche le procedure per le elezioni: il sindacato comunista propone un sistema basato su candidature individuali, che lo avrebbe favorito, data la maggiore esperienza e notorietà dei propri rappresentanti. I socialisti, al contrario, sono per candidature organizzate in liste elettorali. Il governo decide alla fine in favore delle liste elettorali (Foweraker 1987). 
quando il sindacato, o i sindacati, che sottoscrivono l'accordo, controllano più del cinquanta per cento dei rappresentanti di fabbrica nelle rispettive categorie (McElrath 1989, 172).

La relativa autonomia dei sindacati spagnoli dalle autorità di governo può essere meglio apprezzata considerando i livelli di conflitto industriale che prevalgono nei primi anni della transizione. La mobilitazione sindacale in questo paese segue una sequela di fasi considerate ottimali per una solida evoluzione democratica (Valenzuela 1988). In un primo tempo si impone una forte mobilitazione, che permette ai vari segmenti del movimento dei lavoratori di raggiungere, in misura più o meno completa, i propri obiettivi particolari; successivamente prevale un atteggiamento di moderazione, che assicura il compimento del processo di transizione nel suo complesso e permette ai sindacati di consolidare gli obiettivi già raggiunti. I dati riguardanti gli scioperi assumono inizialmente valori piuttosto alti (fino al 1979), ma diminuiscono in seguito, anche in concomitanza con l'introduzione di una più autonoma contrattazione collettiva tra lavoratori ed imprenditori e alla firma di patti socioeconomici di cooperazione tra le parti sociali (Ilo, vari anni).

Sia il primo governo centrista che il successivo governo socialista appoggiano i sindacati, in modo particolare il più moderato sindacato socialista, la Unión general de trabajadores (Ugt). Tra il 1979 ed il 1984, i sindacati (inizialmente solo la Ugt), il governo e gli imprenditori stipulano una serie di accordi generali sui salari, nel contesto di una politica dei redditi intesa a controllare la crescita delle retribuzioni ed a ridurre l'inflazione. In cambio, ai sindacati vengono riconosciuti importanti diritti e una serie di benefici economici e politici, tra i quali misure a sostegno dell'occupazione e una favorevole riforma fiscale. Gli accordi tra le parti vengono facilitati da una regolamentazione della rappresentanza sindacale che permette alle organizzazioni più rappresentative di impegnare all'accordo la quasi totalità dei lavoratori del paese.

Mentre l'appoggio della Ucd si spiega con il tentativo di arginare il più radicale sindacato comunista e si sviluppa in una serie di patti che permette al sindacato socialista di rafforzare progressivamente la sua posizione in seno al movimento dei lavoratori, più organico è il rapporto tra Ugt e Partito socialista. Tale rapporto è documentato sia dallo sviluppo storico comune che dai riferimenti al «partito fratello» negli statuti dell'organizzazione sindacale. Sino a pochi anni fa la Ugt riconosceva espli- 
citamente di essere rappresentata politicamente dal partito ed esprimeva il proprio sostegno ufficiale in suo favore in occasione delle elezioni. La doppia appartenenza, a sindacato e partito, era la norma: la presenza della leadership sindacale nel Psoe, tuttavia, è sempre stata limitata (Fishman 1990, 168-172). Il favore con cui i sindacati sono stati visti, sia dal governo di centro che da quello socialista, è confermato dagli aiuti economici che entrambi hanno elargito con generosità. Il governo socialista ha distribuito ai nuovi sindacati parte del patrimonio della disciolta organizzazione sindacale franchista nel 1985; già precedentemente, comunque, il governo della Ucd aveva disposto ingenti somme a loro favore. Un patto tra i sindacati ed il governo, concluso nel 1981, e rimasto a lungo segreto, disponeva infatti forti pagamenti a favore delle principali organizzazioni sindacali (Domínguez 1988, 76-77). In sintesi, il riuscito consolidamento democratico ha potuto giovarsi, nel caso spagnolo, di una prevalente autonomia del sistema di relazioni industriali e di una continua e pressante opera di mobilitazione, da parte del governo, della più importante e moderata organizzazione sindacale.

In Venezuela il consolidamento democratico, successivo alla transizione politica del 1958, ha avuto un esito sostanzialmente positivo, ma contrassegnato da alcune ombre. Il consolidamento è provato dalle molte alternanze governative avvenute ininterrottamente dal 1958 ad oggi, ma non è stato, e non è, senza problemi. Due colpi di stato vengono portati a termine contro il governo provvisorio durante il primo anno di democrazia. Nel 1960 forze ribelli tentano di rovesciare l'esecutivo. Tra il 1958 ed il 1963 una dozzina di rivolte militari causano più di mille tra morti e feriti. Per di più durante gli anni sessanta si diffonde un forte movimento di guerriglia, che viene sgominato solo dopo molti anni. Più recentemente il paese è stato scosso da altri tentativi di colpo di stato ${ }^{16}$. Alcuni avvenimenti politici e sindacali della fase della transizione sono cruciali per comprendere il successivo sviluppo della democrazia e delle istituzioni di questo paese. Nel 1958, con la crisi della dittatura del generale Pérez Jiménez, i principali partiti politici stipulano un patto, detto di Punto Fijo, che limita il numero dei partiti am-

16 Sulla democrazia in Venezuela si vedano Coppedge (1992); Calcaño Gómez (1992); Karl (1987); e Levine (1973). 
messi alle elezioni, escludendo, tra gli altri, l'importante Partito comunista e fissa regole molto dettagliate sulla spartizione del potere tra vincitori e sconfitti, indipendentemente dai risultati delle imminenti consultazioni elettorali ${ }^{17}$. Una delle conseguenze degli accordi è, come ricordato, la comparsa di un movimento armato clandestino tra i più agguerriti e tenacemente radicati della regione. Le stesse parti siglano un importante patto socioeconomico, l'Avenimiento Obrero-Patronal, nel quale il sindacato si impegna ad evitare il ricorso agli scioperi, ne accetta una disciplina più severa ed approva alcuni accordi collettivi sottoscritti con gli imprenditori ${ }^{18}$.

Il consolidamento del sistema delle relazioni industriali segue un analogo destino di incertezza: il conflitto industriale è contenuto, le rivendicazioni salariali modeste, ma la creazione di un sistema di contrattazione autonomo avviene solo sulla carta. Mentre il diritto alla contrattazione collettiva è sancito nella Costituzione e nella legislazione specifica che ne dà attuazione, le organizzazioni sindacali devono essere riconosciute preventivamente dal governo: questi gode anche di ampia discrezionalità nel sopprimere sindacati già riconosciuti e nel regolare il loro comportamento (Valente 1979, 177-179). L'intervento del governo è particolarmente significativo nell'amministrazione e nella prevenzione dei conflitti industriali: esso si manifesta nei limiti al diritto di sciopero e nell'imposizione di soluzioni obbligatorie alle vertenze industriali sotto la supervisione del potere esecutivo. I dati sul conflitto industriale dimostrano che queste disposizioni hanno favorito un controllo sostanziale degli scioperi, mantenutisi a livelli molto moderati per tutto il corso della transizione ed oltre. Il nuovo regime introduce, tra l'altro, regole che impediscono, o rendono più difficile, l'emergere di organizzazioni sindacali alternative. Si dispone, ad esempio, che solo

17 I partiti più importanti del paese erano: Acción democrática (Ad), di ispirazione vagamente socialdemocratica ed affiliato al sindacato più importante, la Confederación de trabajadores de Venezuela (Ctv); il Partido social-cristiano (Copei) di ispirazione cristiana e la Unión republicana democrática (Urd), più conservatrice (Karl 1987).

18 Più precisamente il patto prevedeva: l'impegno comune di lavoratori e datori di lavoro a rafforzare il nuovo regime democratico; un sistema volontario di conciliazione, stabilito dalle parti, per la risoluzione dei conflitti e la creazione di apposite Comisiones de Avenimiento (Comitati di riconciliazione); la promessa dei datori di lavoro di limitare i licenziamenti; l'obbligo di osservare gli accordi collettivi sottoscritti e, più in generale, il vigente diritto del lavoro; l'introduzione di procedure che sottoponevano l'esercizio del diritto di sciopero a controlli e restrizioni (López Maya 1989; Karl 1987, 63 95; Godio 1986). 
una maggioranza di lavoratori in ogni settore di produzione possa eleggere propri rappresentanti, favorendo di gran lunga la diffusione del sindacato governativo, meglio organizzato e con maggiori risorse. Il consenso dei lavoratori alla contrattazione collettiva, inoltre, è nominale. I leader dei maggiori partiti esercitano, infatti, un controllo ferreo sul sindacato: essi scelgono direttamente $\mathrm{i}$ loro dirigenti ed elaborano i programmi politici ed economici dell'organizzazione, senza la partecipazione ed il consenso della base. Le più importanti decisioni e scelte programmatiche si riducono spesso all'approvazione di programmi già definiti. La Confederación de trabajadores de Venezuela (Ctv), insomma, si dimostra perennemente incapace di elaborare progetti politici originali, diversi da quelli del governo o degli imprenditori. Il grado di dipendenza è tale che la stessa contrattazione collettiva non prevede, spesso, la partecipazione o la presenza dei lavoratori, i quali sono informati degli accordi solo al momento della firma (Godio 1982, 43-49). L'intrusione del potere politico nella conclusione degli accordi collettivi e nelle procedure per la soluzione dei conflitti del lavoro, in sintesi, indicano i limiti dell'istituzionalizzazione delle relazioni industriali e della stessa democrazia in Venezuela: il prezzo pagato per un consolidamento politico, conseguito con mezzi relativamente non democratici, si riflette nella limitata autonomia concessa ai gruppi di interesse e in una ridotta legittimità del sistema.

I governi, per contro, assicurano ingenti finanziamenti. Contrariamente a quanto spesso sostenuto dalla propaganda sindacale, i contributi governativi costituiscono il reddito principale dell'organizzazione: nei primi anni sessanta, i fondi del governo rappresentano il 90 per cento delle entrate sindacali. Negli anni successivi alla transizione l'esecutivo si è impegnato in una serie di opere a favore dei sindacati, tra cui la costruzione di alcune sedi e la creazione di una banca dei lavoratori, usata però dal sindacato per gestire un vasto network di imprese ed operare ambigue operazioni di credito a loro favore (Godio 1982, 94). Gli stretti rapporti esistenti tra la Ctv e i maggiori partiti del paese hanno favorito una continua e costante provvigione di risorse finanziarie ed organizzative. Acción democrática ha a lungo esercitato una posizione di predominanza sul sindacato: se nel 1959 più della metà dei delegati sindacali appartenevano a questo partito, solo pochi anni più tardi essi raggiungevano il settanta per cento, mentre un altro terzo era appannaggio degli altri due partiti principali, ad esso alleati. La 
doppia affiliazione degli iscritti al sindacato e dei rappresentanti dei partiti di governo (in modo particolare Ad) è stata comune. I rappresentanti sindacali in Acción Democrática erano organizzati in un Buró Sindical, che non ha mai esercitato alcun potere nel partito e che si è dimostrato incapace di rappresentare e difendere gli interessi dei lavoratori in sede politica (López Maya e Gómez Calcaño 1985). La vicinanza, soprattutto con Acción Democrática, è dimostrata anche dal diverso atteggiamento sindacale nei confronti dei governi in carica: durante le amministrazioni di Ad le rivendicazioni salariali e le manifestazioni di dissenso sono state più contenute.

L'inclusione del sindacato in una coalizione di governo particolarmente stabile, pur avallando una situazione di dubbia legittimità democratica, permette una politica di moderazione dei salari e del conflitto sindacale, facilita la manovra macroeconomica del governo ed aiuta la stabilizzazione ed il rafforzamento del nuovo regime. La particolare architettura istituzionale, assieme ad interventi di repressione dei sindacalisti dissenzienti e ad ulteriori misure di controllo politico, consentono l'appoggio del sindacato a tutte le maggiori iniziative politiche ed economiche del governo. I limiti posti alla partecipazione elettorale, in modo particolare l'esclusione della sinistra più militante, impediscono che il malcontento, diffuso tra le fila sindacali, si tramuti in alternativa politica organizzata e consentono ai partiti al potere di perseverare nelle loro strategie ${ }^{19}$.

È generale il consenso attorno all'avvenuto consolidamento democratico in Uruguay (Gunther, Diamandouros e Puhle 1995, 19; Mainwaring 1994, 10). Il processo di rafforzamento

19 Una simile configurazione dei rapporti tra sindacati, partiti e stato esiste in Messico, Paraguay, Cile e Costa Rica. Nei primi due casi la stabilità politica, compromessa dall'eccessiva ingerenza dello stato nelle relazioni tra le parti sociali, è meno significativa, per il carattere autoritario dei regimi politici, ma rappresenta ugualmente una minaccia alla loro sopravvivenza. In Cile l'instabilità politica è temperata dalla debolezza relativa del sindacato, che è anche strettamente connessa al movimento democratico, di cui, almeno per ora, rispetta le direttive di moderazione politica e salariale. Il gap di legittimità, derivante dall'imposizione eteronoma di regole e norme per la disciplina delle relazioni industriali è così parzialmente e momentaneamente ridotto dal carattere prevalentemente politico dei rapporti tra sindacato ed imprenditori che sono, in questa fase, interessati a mantenere e rafforzare il nuovo regime democratico. In Costa Rica, infine, la considerevole stabilità politica di cui gode il nuovo regime democratico e che contraddice il modello esplicativo qui proposto, è giustificata dalla particolare debolezza del movimento dei lavoratori e dalla sua peculiare, recente affiliazione ai datori di lavoro. 
delle istituzioni e pratiche democratiche ha avuto i suoi punti di forza in una costituzione che ha sancito, tra le prime nel continente, la possibilità del suffragio maschile, diretto e segreto ed in un sistema partitico particolarmente solido (Hartlyn e Valenzuela 1995,19$)^{20}$. Fondamentale, a questo proposito, anche il raggiungimento dell'accordo del Club Nautico, siglato tra governo militare ed opposizione nell'agosto 1984 . Nel patto, l'opposizione civile promette ai militari che non saranno prese misure punitive nei loro confronti. I militari, a loro volta, permettono il ritorno all'ordine costituzionale precedente, con elezioni libere programmate per il novembre dello stesso anno. Uno degli aspetti significativi dell'accordo è la partecipazione del Frente amplio, una nuova formazione politica di sinistra, che obbliga $\mathrm{i}$ militari a ricercare un accordo che sia accettabile per la quasi totalità degli uruguayani. Successivamente i governi democratici hanno sempre cercato ampie forme di cooperazione con l'opposizione, che ha spesso collaborato, anche direttamente, alle responsabilità politiche ed amministrative dell'esecutivo.

Nonostante il raggiunto consolidamento politico, tuttavia, si riconoscono, in modo ugualmente unanime, una serie di problemi che hanno contribuito a limitarne il significato pratico. La severa crisi economica affrontata dal paese e la scarsa efficacia delle politiche governative hanno provocato una generale insoddisfazione, che si è manifestata soprattutto in una intensissima serie di conflitti industriali e manifestazioni di protesta. Durante tutto il 1985 e sino al 1987 vengono proclamati più di mille scioperi, che coinvolgono soprattutto i lavoratori pubblici (Bronstein 1995, 201). È durissima anche l'opposizione al piano governativo di concedere un'amnistia per i crimini commessi dai militari durante il precedente governo autoritario, che viene però confermato $\mathrm{da}$ un referendum popolare. Il conflitto si intensifica ancora attorno alle elezioni del 1989 e si inasprisce ulteriormente durante il successivo governo Lacalle, esponente del Partido Blanco: dal marzo 1990 all'agosto 1992 la Confederazione sindacale uruguayana dichiara ben dieci scioperi generali contro le politiche del governo. La popolarità dei nuovi

20 In Uruguay i presidenti hanno governato nel rispetto delle opposizioni, senza indebolire sistematicamente, o raggirare, il parlamento né altri meccanismi di controllo democratico. Il parlamento e il potere giudiziario contano, i presidenti devono venire a patti con il congresso e con alcuni attori sociali ed hanno, infine, evitato gli eccessi del «decretismo», comuni in Argentina e Brasile (Mainwaring 1994, 34). 
presidenti democratici, Sanguinetti e Lacalle, infine, rimane costantemente bassa (Mainwaring 1994, 38). Il consolidamento democratico in Uruguay, insomma, pur considerevole, non è stato, e non è, privo di problemi: in particolare rimane alta la conflittualità sociale e specialmente quella del lavoro.

Il caso uruguayano è peculiare anche da un punto di vista sindacale. L'Uruguay, infatti, non è solo uno dei pochi paesi della regione a vantare una Confederazione sindacale unica, il Plenario intersindical de trabajadores - Convención nacional de trabajadores (Pit-Cnt), ma rappresenta il solo caso latino-americano di sostanziale autonomia delle relazioni industriali dallo stato nazionale, sia a livello giuridico che nella prassi vigente. In una delle prime misure adottate durante la transizione democratica, il governo ha annullato e dichiarato inesistente la legislazione sulle relazioni collettive di lavoro promulgate dal regime militare. Questo intervento, che non è stato seguito dalla adozione di nuove norme, ha fatto ritornare il paese al suo peculiare sistema di autoregolamentazione o, se si preferisce, di deregolamentazione delle relazioni industriali. Scarse sono le regole che disciplinano o restringono il diritto di formare un sindacato o di proclamare uno sciopero e i governi democratici si sono dimostrati tolleranti nei confronti della pur elevata militanza sindacale (Bronstein 1995, 195; Berins Collier e Collier 1991, 652-656). La preponderanza dell'economia di Montevideo nella piccola nazione del Cono Sur ha permesso di sviluppare un'economia relativamente omogenea e avanzata e quindi sindacati organizzati a livello di settore, mentre la disposizione spesso benevola o neutrale dei governi preautoritari nei confronti dei lavoratori e la capacità di mobilitazione del sindacato possono dar conto della maggiore forza e considerazione goduta e dell'ampia autonomia nei confronti dello stato.

Anche nei rapporti con i partiti politici prevale una fondamentale autonomia. La tradizione anche in questo caso, è favorevole: nonostante gli sforzi del Partito comunista, tesi alla unificazione del movimento sindacale, questo è rimasto a lungo diviso ed ha mantenuto una marcata indipendenza nei confronti dei partiti tradizionali e dello stesso Partito colorado, che pure aveva contribuito agli enormi miglioramenti salariali e nello standard di vita realizzati negli anni quaranta (Berins Collier e Collier 1991, 653). Se una certa predominanza del Partito comunista perdura durante i primi anni del nuovo regime democratico, si mantiene una radicata indipendenza sindacale, so- 
prattutto dai partiti di un certo peso, organizzati nel blocco maggioritario di natura centrista che di fatto guida il paese (Berins Collier e Collier 1991, 503). L'autonomia del sindacato, anzi, sarebbe tale da farlo agire, spesso, come un vero e proprio movimento politico (Boschi 1990, 224). Il sindacalismo uruguayano cercherebbe, insomma, di esercitare un'influenza diretta nel conflitto con le classi medie, accusate di essere responsabili della crisi e di accettare una dipendenza crescente nei confronti del sistema economico e finanziario internazionale (Touraine 1988, 136).

La mancanza di una forte tradizione corporativa e la peculiare natura dei rapporti con i partiti politici conferiscono un carattere unico al processo di consolidamento in Uruguay, caratterizzato dalla già ricordata estrema conflittualità sociale ed economica, ma nel contesto di istituzioni e pratiche politiche saldamente democratiche. Pure nel quadro di un giudizio complessivamente favorevole alle prospettive di consolidamento, tuttavia, non possono essere sottovalutati i potenziali pericoli che sul corso ulteriore del mantenimento democratico finiscono col produrre le continue manifestazioni di protesta industriale e di ribellione sociale. La sostenuta ed incisiva militanza industriale è da numerosi osservatori ritenuta una tra le cause più significative della progressiva militarizzazione della politica, che, durante i primi anni settanta, ha fatto precipitare il paese nel baratro dell'esperienza autoritaria (Berins Collier e Collier 1991, 505) ${ }^{21}$.

La Bolivia, infine, rappresenta l'epitome dell'instabilità politica ed un caso di consolidamento non risolto. Nella prima metà degli anni ottanta è presidente Siles Zuazo (1982-1985), sostenuto da una coalizione composta da due partiti di stampo populista (il Movimiento nacionalista revolucionario de izquier$\mathrm{da}$, cui appartiene il presidente, ed il Movimiento de la izquierda revolucionaria del vice-presidente Jaime Paz Zamora) e dal debole Partito comunista ${ }^{22}$. Scossa da continue crisi interne,

21 Un caso, in parte simile, è quello dell'Argentina, dove la precaria mobilitazione del movimento sindacale è stata accompagnata da una relativa autonomia dal potere di regolamentazione dello stato. Oltre alla possibilità di crisi economiche, come in Uruguay, sussiste dunque in questo paese il pericolo di instabilità politica connessa alla militanza di un sindacato spesso direttamente controllato e regolato dallo stato.

22 A scapito dei nomi, alquanto bellicosi, questi partiti sono di ideologia nazionalista e moderata, pur rappresentando gli eredi politici del Movimiento nacionalista revo- 
fronteggiata da un parlamento controllato dall'opposizione e costretta a decidere sistematicamente per decreto, la coalizione vacilla tra interventi economici di stabilizzazione approvati dal Fondo monetario internazionale e concessioni ai gruppi sociali più potenti, che vanificano ogni sforzo di razionalizzazione economica. Tra gli avvenimenti politici che caratterizzano questo periodo vi sono diversi tentativi di colpi di stato, il frequente rifiuto delle Forze armate di dare esecuzione a ordini e provvedimenti del presidente, il suo rapimento da parte di militari ribelli, nonché diversi tentativi di «golpe costituzionale» ad opera dei partiti rappresentati in parlamento. Le relazioni con il potente sindacato boliviano, la Central obrera boliviana (Cob), si dimostrano subito molto difficili: durante i primi tre mesi della presidenza Siles vengono proclamati 204 scioperi. Il confronto con il sindacato vive il suo culmine nel febbraio 1985, quando, dopo l'ennesima approvazione di misure economiche restrittive, migliaia di minatori circondano la città di $\mathrm{La} \mathrm{Paz}$ e dichiarano uno sciopero generale. Il governo, esasperato, invia l'esercito che rompe l'assedio e costringe i sindacati ad accettare le proprie proposte (Malloy e Gamarra 1988, 161). Successivamente una serie di patti politici tra i principali partiti di destra e di centro permette un certo rafforzamento del processo di democratizzazione, che però appare lungi dall'essere risolto. In particolare, scioperi generali e manifestazioni contro le politiche di austerità dei nuovi governi si sono moltiplicati sino ad oggi. Come in precedenza, il nuovo governo ha reagito imponendo, in diverse occasioni, lo stato d'assedio e persino l'occupazione militare della capitale.

Nel settore pubblico, strategico per l'economia del paese, lo stato ha svolto e di fatto continua a svolgere, un'azione globale nella trattativa salariale, assumendo il ruolo di parte sociale fondamentale, con scarsi effetti di istituzionalizzazione del sistema ${ }^{23}$. Nel settore privato e regolato dell'economia, invece, la contrattazione si svolge interamente a livello della singola impresa. Sebbene introdotto già nel 1942, il contratto collettivo trova un'effetti-

lucionario, il partito che ha messo fine al potere delle élites oligarchiche dei grandi proprietari terrieri e minerari del paese con la rivoluzione popolare del 1952.

23 In questo ambito deve essere ricordata anche la pratica, diffusa sino al 1982, della partecipazione del sindacato a governi ed enti di amministrazione e gestione delle risorse pubbliche, tra i quali ha particolare importanza il Comibol, l'impresa mineraria boliviana. 
va utilizzazione solo in alcuni settori, come quello minerario e dei trasporti ferroviari, nei quali i sindacati sottoscrivono accordi su temi economici e sociali (Blanes 1993, 310). Questi fattori e il ridimensionamento del potere contrattuale del sindacato, dovuto alla drammatica riduzione dell'occupazione nei settori ad alta concentrazione sindacale, come quello minerario, non devono però dare la falsa impressione di un sistema regolato dal mercato, in cui l'ingerenza statale sia trascurabile. Prima del 1985 lo stato mediava e regolava ogni tipo di conflitto di lavoro ${ }^{24}$. Anche successivamente, tuttavia, nonostante alcune riforme in senso liberista, lo stato ha continuato a gestire gli ambiti più importanti della contrattazione economica, intervenendo in maniera diretta e a volte violenta per reprimere scioperi e manifestazioni di protesta, soprattutto nei settori cruciali delle miniere e dell'educazione.

Il potente sindacato boliviano è stato caratterizzato, almeno recentemente, dalla mancanza di significativi legami organizzativi con importanti partiti politici. Spezzati i rapporti con il Movimiento revolucionario nacionalista $(\mathrm{Mnr})$ e superata la fase di partecipazione ai nuovi governi democratici, la Cob si radicalizza e si avvicina a ideologie marxiste-leniniste rivoluzionarie, i cui orientamenti si risolvono spesso in uno scontro diretto con lo stato. In questa fase il sindacato esercita una vera e propria funzione di controllo su alcuni partiti di estrema sinistra, la cui limitata rilevanza elettorale, tuttavia, riduce l'importanza complessiva dell'azione sindacale (Grassi 1993) ${ }^{25}$. Durante la recente democratizzazione, abbandonate in gran parte le aspirazioni socialiste, il sindacato persegue l'obiettivo più limitato di rafforzare ed imporre il proprio potere (Touraine 1988).

Nonostante la prevalente immagine di unità sindacale e di un'ampia protezione dei diritti dei lavoratori, nell'ambito della

24 È solamente in un secondo momento, dopo il 1985, che il nuovo governo conservatore abbandona questa strategia ed adotta un approccio più indiretto con cui diminuisce il potere del sindacato e riafferma un sistema basato sulla contrattazione individuale e sul mercato. Questa alternativa ha il vantaggio di presentare la nuova situazione come il semplice manifestarsi di forze oggettive operanti al di fuori del controllo dell'esecutivo, che evita quindi il biasimo per l'adozione di misure repressive poco popolari.

25 Il Partito comunista boliviano si assicura il controllo del sindacato durante il V Congresso della Cob, celebrato nel 1979. Cinque anni più tardi il controllo passa ad una coalizione di piccoli partiti marxisti-leninisti, organizzati in una Dirección revolucionaria unificada (Dru), favorevoli ad uno scontro aperto con il governo per l'instaurazione di un regime di tipo socialista. Nel 1987, infine, il Partito comunista ritorna a guidare il grande sindacato, ottenendo un ammorbidimento delle sue tesi più estreme. 
disciplina legale delle relazioni industriali prevalgono i particolarismi mentre la capacità di apportare miglioramenti alle condizioni della massa dei lavoratori si è rivelata marginale ${ }^{26}$. La disciplina normativa e la regolamentazione dell'ordinamento dei rapporti tra sindacati e datori di lavoro non si fonda, in altre parole, su di un sistema che garantisce a tutti i lavoratori gli stessi diritti, ma piuttosto su di uno sviluppo di gruppi e settori particolari, che, a seconda della rispettiva forza, sono stati capaci di ottenere certi vantaggi e prerogative. Un esempio è costituito dal sistema della previdenza sociale: la strategia sindacale è stata quella di lotte ed obiettivi settoriali, condotti a seconda delle rispettive forze da ciascun sindacato ed il risultato quello del proliferare di istituti locali e fondi speciali. Durante la presidenza di Siles Zuazo si accentuano questi caratteri: la lotta sindacale, concentrata sul salario, produce una ulteriore atomizzazione dei conflitti, il cui grado di istituzionalizzazione raggiunge i livelli più bassi della storia del sindacalismo boliviano (Blanes 1993, 311). Il conflitto, nei suoi diversi indicatori, si attesta a livelli molto alti, che dimostrano come l'esplosione degli scioperi non sia mediata da istituzioni capaci di creare spazi di soluzione dei conflitti in un ambito democratico e rappresentativo ${ }^{27}$.

\section{Conclusioni}

Si possono a questo punto meglio valutare le ipotesi alternative illustrate all'inizio. L'interpretazione qui proposta ha punti

26 In aggiunta alla Costituzione del 1967 che tutela alcuni diritti basilari dei lavoratori, è da ricordare la prima Legge del Lavoro, del 1939, cui sono state apposte ben 2.500 disposizioni complementari, espresse in almeno 500 leggi, decreti e risoluzioni ministeriali (Blanes 1991, 21).

27 Un esito simile a quello boliviano dovrebbe prevalere, data la configurazione del rapporto tra stato, partiti ed attori sociali, in Ecuador, Perù e Brasile, ma anche in Colombia. In questi casi, infatti, il sindacato non è stato politicamente mobilitato e lo stato ha esercitato un controllo stretto sui rapporti industriali e sulla contrattazione tra le parti. Di conseguenza sia la stabilità politica che quella economica di questi regimi dovrebbero essere particolarmente precarie, come dimostra appunto la crisi autoritaria in corso in Perù. In Colombia, nella fase di transizione alla democrazia, la prevalenza di élites economiche legate alla oligarchia tradizionale favorisce forme di controllo e smobilitazione dei lavoratori attraverso i partiti politici già esistenti, che hanno con i sindacati legami solo marginali. La relativa debolezza dei sindacati e l'efficace incorporazione politica attraverso i partiti giustificano la maggiore stabilità sperimentata dal paese sin dagli anni cinquanta, ma recentemente entrata in crisi. Una breve nota su Portogallo e Grecia: entrambi i paesi sono stati caratterizzati da scarsa autonomia sindacale e da una situazione di parziale mobilitazione che ha prodotto una relativa stabilità economica ed una maggiore instabilità politica, che solo con il tempo si è andata affievolendo. 
in comune con alcune ipotesi di carattere storico. A differenza di queste, tuttavia, l'attenzione è in questo saggio rivolta ai più recenti processi di democratizzazione in America Latina ed Europa del Sud, che hanno avuto luogo in condizioni economiche e politiche molto diverse. Le nuove democrazie latinoamericane e sudeuropee si affermano e sopravvivono in un ambiente economico ostile, che si manifesta in politiche di esclusione e marginalizzazione sociale ed in una crisi organizzativa e numerica dei sindacati e delle organizzazioni sociali. Le forme della democratizzazione e della mobilitazione sindacale riflettono le strategie politiche delle élites al potere al momento della transizione e spiegano le modalità e le specie dell'inclusione ed emarginazione dei sindacati nei nuovi regimi politici. La mobilitazione dei lavoratori come importante risorsa politica, o la loro smobilitazione, producono contrastanti reazioni e controreazioni, che pongono le fondamenta per distinti modelli di consolidamento democratico. Da un lato, come ho indicato, si pone l'esigenza di creare istituzioni per regolare il conflitto industriale e mantenere sotto controllo la militanza sindacale. Dall'altro c'è il problema della legittimità del nuovo regime democratico, che deve godere di una base sociale e di un sostegno politico sufficientemente forti per garantirne la sopravvivenza e la maturazione.

Il punto, ovviamente, non è quello di negare l'importanza dei fattori socioeconomici come determinanti di alcuni fenomeni politici, ma quello di sottolineare, come si è già ricordato, che il loro impatto non è così diretto come parte della letteratura vorrebbe. Alcuni cambiamenti economici hanno costituito una significativa parte del contesto nel quale ebbero luogo le trasformazioni politiche qui analizzate, ma la loro rilevanza causale è stata spesso esagerata. L'impatto dei cambiamenti socioeconomici è a volte relativamente chiaro, come nel caso dello sviluppo del commercio mondiale, nella seconda metà del secolo scorso e nei primi decenni di quello successivo, che promuove la crescita delle esportazioni, una maggiore urbanizzazione e l'espansione dell'industria manufatturiera, fenomeni che, a loro volta, creano nuovi attori sociali e politici. Altre volte è più indiretto, come nel rapporto tra i cambiamenti appena citati e l'emergere del movimento dei lavoratori. Altre volte ancora, infine, è ambiguo, come nell'effetto delle depressioni economiche sui rapporti tra governo e sindacati e sui processi di democratizzazione e consolidamento democratico.

Anche le variabili organizzative, di per sé, non possono dar 
conto dei diversi esiti dei processi in esame. Nonostante la loro diversità, infatti, i sindacati di cui si è discusso sono stati caratterizzati, con la possibile eccezione dell'Uruguay, da una sostanziale identità di forze ${ }^{28}$. In Argentina, per citare un altro caso rilevante, il livello di sindacalizzazione subito dopo la transizione è simile a quello spagnolo nel periodo corrispondente (in entrambi i casi si aggira sul 35 per cento della forza lavoro dipendente). Pur essendo durante questo periodo più unito, e quindi teoricamente più forte, il sindacato argentino non conclude però accordi specifici con il governo e gli imprenditori e mantiene livelli elevati di conflittualità, contrariamente a quanto ipotizzato dalle prevalenti teorie organizzative. Ancora più evidente è il caso del sindacato boliviano, unito e relativamente forte, i cui rapporti con il governo in carica sono caratterizzati da una conflittualità esasperata che conduce rapidamente ad uno scontro generalizzato (Dunkerley 1989; Lazarte 1990) ${ }^{29}$. Più importanti appaiono i riferimenti di questa letteratura alla moderazione ideologica e soprattutto all'affiliazione politica con i partiti. Anche in questo caso, tuttavia, si deve osservare che queste variabili, utilizzate in questo ambito esclusivamente come variabili «indipendenti», cioè come elementi causali nel processo esplicativo, devono essere, invece, a loro volta giustificate. In questo senso, l'interpretazione proposta è più completa, perché dà ragione delle affiliazioni organizzative e delle affinità ideologiche, che dipendono, come si è visto, dai progetti politici e dalle dinamiche interne delle élites che presiedono ai processi di transizione e consolidamento.

Questi elementi suffragherebbero dunque la conclusione che i nuovi regimi democratici hanno tratto numerosi benefici dall'istituzionalizzazione di un sistema di relazioni industriali, in particolare un più completo consolidamento democratico ed una accresciuta stabilità politica. Una eccessiva preoccupazione per l'aspetto della stabilità, tuttavia, ha qualche volta diminuito il significato dell'inclusione sindacale, ridotta a mera formalità:

28 La minore affiliazione sindacale in Uruguay è compensata dall'unità del movimento sindacale e da una notevole capacità di mobilitazione, che fa figurare il movimento sindacale di questo paese tra i più forti dell'America Latina (Ilo 1995, 67).

29 Il sindacato venezuelano ha, alla fine degli anni cinquanta, una forza simile a quella del sindacato della vicina Colombia, ma gli esiti politici del rapporto con i rispettivi sistemi politici è radicalmente diverso, traducendosi in una inclusione politica nel primo caso ed in una sua sostanziale esclusione nel secondo. 
in questo caso, gli effetti positivi hanno teso ad affievolirsi col tempo, impedendo un consolidamento duraturo e permanente del nuovo regime, che è rimasto esposto all'azione destabilizzante di crisi economiche e sociali, cui il nuovo regime non ha saputo o potuto fornire i canali per un'espressione sistematica e non cruenta.

Alcuni recenti cambiamenti di carattere più generale, inoltre, hanno aggiunto ulteriori aspetti problematici a questi processi. Il trionfo delle politiche neoliberali in America latina e nel resto del mondo si è realizzato attraverso misure come l'apertura delle economie al mercato internazionale, la privatizzazione delle imprese statali, la fine del corporativismo sindacale, ed altre ancora, dirette ad aumentare la competitività delle imprese nazionali. A queste si sono accompagnati un profondo mutamento della struttura occupazionale e delle forme di produzione, (mobilità delle imprese, precarizzazione e subcontrattazione), la crescente pauperizzazione dei lavoratori dipendenti ed una crisi profonda dei rapporti tra questi settori ed i partiti politici, che tendono sempre più ad escludere una loro rappresentazione diretta e di cui spesso non ricercano più l'appoggio (Zapata 1993). Questi processi hanno intaccato in modo visibile le basi legali e sociali delle relazioni industriali e, se l'analisi fin qui svolta è adeguata, hanno contribuito ad aggravare la crisi di governabilità dei nuovi regimi democratici, anche se hanno risolto alcuni aspetti immediati di stabilità ${ }^{30}$. Le regolazioni di tipo associativo-collaborativo costituirebbero infatti uno strumento particolarmente opportuno di consolidamento della democrazia. Esse contribuirebbero a tenere sotto controllo o ad impedire il ciclo politico degli affari e potrebbero bloccare il ciclo democrazia-autoritarismo o ridurne drasticamente la portata e rilevanza politico-sociale (Cella 1990, 24). Le soluzioni alla duplice difficoltà, sindacale e politica, appaiono problematiche: sia che si facciano strada nuove organizzazioni sindacali tese alla rappresentanza di movimenti sociali più vasti (Munck 1994), sia che si scoprano nuove forme di partecipazione politica diretta e nuove ideologie (Zapata 1993), molto sembra di-

30 Un sostegno indiretto alle tesi qui presentate è fornito dai lavori sul rapporto tra neocorporativismo nei paesi industrializzati, da un lato, e livelli di conflitto sociale e reddito, dall'altro. Secondo queste ricerche, laddove ha prevalso un sistema corporativo forte sono stati più contenuti gli scioperi ed il conflitto industriale e maggiore la crescita del reddito nazionale (Cameron 1984; Lange 1984). 
pendere dalla capacità dei sindacati di scegliere vantaggi più permanenti, collegati però all'apertura di un ciclo economico e politico a lungo termine, piuttosto che ricompense immediate ma solo temporanee.

Riferimenti bibliografici

Bergquist, C. (1986), Labor in Latin America: Comparative Essays on Chile, Argentina, Venezuela and Colombia, Stanford, Stanford University Press.

Berins Collier, R. e D. Collier (1991), Shaping the Political Arena. Critical Junctures, the Labor Movement, and Regime Dynamics in Latin America, Princeton, Princeton University Press.

Berins Collier, R. e J. Mahoney (1997), Adding Collective Actors to Collective Outcomes, in «Comparative Politics», n. 2, pp. 285-303.

Blake, C.H. (1994), Social Pacts and Inflation Control in New Democracies, in «Comparative Political Studies», XXVII, 2, pp. 381401.

Blanes, J. (1993), El mercado de trabajo boliviano: del enfrentamento directo a la negociación, in Restructuración y regulación institucional del mercado de trabajo en América Latina, Ginevra, a cura dell'Instituto Internacional de Estudios Laborales, pp. 302-322.

Boschi, R.R. (1990), Social Movements, Party System and Democratic Consolidation: Brazil, Uruguay and Argentina, in Ethier (a cura di), pp. 214-234.

Bronstein, A.S. (1995), Cambio social y relaciones de trabajo en América Latina: balance y perspectivas, in «Revista Internacional del Trabajo», CXIV, 2, pp. 185-209.

Buchanan, P.G. (1991), Reconstituting the Institutional Bases of Consent: Notes on State-Labor Relations and Democratic Consolidation in the Southern Cone, Working Paper n. 160, The Hellen Kellogg Institut for International Studies, Notre Dame, University of Notre Dame.

Calcaño Gómez, L. (1992), Venezuela: perte de légitimité démocratique et coup d'état, in «Problèmes d'Amérique Latine», n. 6, pp. 3-42.

Cameron, D. (1984), Social Democracy, Corporatism, Labor Quiescence and the Representation of Economic Interests in Advanced Capitalist Societies, in Goldthorpe (a cura di), pp. 143-178.

Cella, G. (1990), Debolezze del pluralismo in America Latina. Quali possibilità per la concertazione sociale?, in «Stato e mercato», n. 28, pp. 3-27.

- (1995), Sindacalismo e Società in America Latina, in «Sociologia del Lavoro», n. 55, pp. 7-13. 
Collier, D. (1979) (a cura di), The New Authoritarism in Latin America, Princeton, Princeton University Press.

Coppedge, M. (1992), Venezuela's Vulnerable Democracy, in «Journal of Democracy», n. 4, pp. 32-44.

Cordova, E. (1985), Pactos Sociais: expêriencia internacional, tipologia e modelos, San Paolo, Ibrart.

Cuevas, A. (1986), Democrazia e sviluppo: problemi di consolidamento democratico in America Latina, Roma, Edizioni del Lavoro.

Domínguez, J. (1988), Diez años de relaciones industriales en España, in A. Zaragoza (a cura di), Pactos sociales, sindicatos y patronal en España, Madrid, Siglo XXI, pp. 75-105.

Dos Santos, M. (a cura di) (1985), Concertación politico-social y democratización, Buenos Aires, Clacso.

Dunkerley, J. (1989), Political Transitions and Economic Stabilization: Bolivia, 1982-1989, London, Institute of Latin American Studies.

Epstein, E.C. (a cura di) (1989), Labor Autonomy and the State in Latin America, Boston, Unwin Hyman.

Ermida Uriarte, O. (1993), La intervención estatal en las relaciones colectivas de trabajo latinoamericanas, in «Nueva Sociedad», n. 128, pp. 29-37.

Ethier, D. (a cura di) (1990), Democratic Transitions and Consolidations in Southern Europe, Latin America and Southeast Asia, London, Mcmillan.

Fishman, R.M. (1990), Working Class Organization and the Return to Democracy in Spain, Ithaca, Cornell University Press.

Foweraker, J. (1987), Corporatist Strategies and the Transition to Democracy in Spain, in «Comparative Politics», XX, 3, pp. 57-72.

Godio, J. (1982), El movimiento obrero venezolano, Caracas, Editorial Ateneo de Caracas.

- (1986), 50 años de la CTV. 1936-1986: bistoria doctrina y acción, Caracas, Editorial Nueva Sociedad.

Goldthorpe, J.H. (a cura di) (1984), Order and Conflict in Contemporary Capitalism, Oxford, Oxford University Press.

Grassi, D. (1993), Socioeconomic Pacts During Transitions to Democracy, Ph.D. dissertation, Chicago, University of Chicago.

Gunther, R., P.N. Diamandouros e H.J. Puhle (a cura di) (1995), The Politics of Democratic Consolidation: Southern Europe in Comparative Perspective, Baltimore, The Johns Hopkins University Press.

Hartlyn, J. e A. Valenzuela (1995), La democracia en America Latina desde 1930, in «Este País, Folios», n. 46, pp. 1-40.

Hirschman, A. (1979), The Turn to Authoritarianism in Latin America and the Search for its Economic Determinants, in D. Collier (a cura di), The New Authoritarianism in Latin America, Princeton, Princeton University Press, pp. 61-98.

Karl, L.T. (1987), Petroleum and Political Pacts: the Transition to De- 
mocracy in Venezuela, in «Latin American Research Review», XX, 1, pp. 63-94.

International Labor Organization (Ilo) (vari anni), El Trabajo en el Mundo, Ginevra, Ilo.

- (1996) La consulta tripartita en el plano nacional sobre la política económica y social, Ginevra, Ilo.

- (vari anni), Yearbook of Labor Statistics, Ginevra, Ilo.

Lange, P. (1984), Unions, Workers and Wage Regulation: the Rational Bases of Consent, in Goldthorpe (a cura di), pp. 98-123.

Lazarte, J.R. (1990), Movimientos Obreros y procesos politicos en Bolivia: hystoria de la COB (1952-1987), La Paz, Edobol.

Lechner, N. (1985), Pacto Social nos processos de democratização: a experiência Latino-Americana, in «Novos Estudos», XXIII, 1, pp. 29-44.

Levine, D.H. (1973), Conflict and Political Change in Venezuela, Princeton, Princeton University Press.

Linz, J.J. (1990), Transitions to Democracy, in «Washington Monthly», n. 13 , pp. 143-164.

López Maya, M. (1989), De Punto Fijo al pacto social: desarrollo y begemonía en Venezuela, 1958-1985, Caracas, Fondo Editorial Acta Cientifica Venezolana.

López Maya, M. e L. Goméz Calcaño (1985), Crisis y concertación en Venezuela: dos coyunturas históricas, in Dos Santos (a cura di).

Mainwaring, S. (1994), Democracy in Brazil and the Southern Cone, Achievements and Problem, Notre Dame, The Helen Kellogg Institute for International Studies, Project Latin America 2000 Series, working paper n. 2.

Malloy, J. e E. Gamarra (1988), Revolution and Reaction: Bolivia, 1964-1985, Oxford, Transaction Books.

Maravall, J.M. (1982), The Transition to Democracy in Spain, London, Croom Helm.

McElrath, R. (1989), Trade Unions and the Industrial Climate in Spain, Philadelphia, University of Pennsylvania Press.

Morlino, L. (1986), Consolidamento democratico: definizione e modelli, in «Rivista Italiana di Scienza Politica», n. 2, pp. 197-236.

Munck, G.L. (1994), Democratic Transitions in Comparative Perspecti$v e$, in «Comparative Politics», n. 3, pp. 355-375.

O'Donnell G. (1977), Corporatism and the Question of the State, in J.Malloy (a cura di), Authoritarianism and Corporatism in Latin America, Pittsburg, University of Pittsburg Press, pp. 47-87.

- (1979), Tensions in the Burocratic-Authoritarian State and the Question of Democracy, in D. Collier (a cura di), The New Authoritarianism in Latin America, Princeton, Princeton University Press, pp. 285-318.

- (1992), Delegative Democracy?, Working paper n. 172, The Hellen Kellogg Institute for International Studies, Notre Dame, Notre Dame University Press. 
O'Donnell, G. e Ph.C. Schmitter (1986), Negotiating (and Renegotiating) Pacts, in G. O'Donnell, Ph.C. Schmitter e L. Whitehead (a cura di), Transitions from Authoritarian Rule: Prospects for Democracy, Baltimore, The Johns Hopkins University Press, pp. 37-47.

- (1986), Transitions from Authoritarian Rule: Tentative Conclusions about Uncertain Democracies, Baltimore, The Johns Hopkins University Press.

Payne, J. (1965), Labor and Politics in Peru, New Haven, Yale University.

Pérez-Díaz, V. (1986), Economic Policies and Social pacts in Spain during the Transition: the Two faces of Neocorporatism, in «European Sociological Review», n. 1, pp. 1-19.

- (1993), The Return of Civil Society: The Emergence of Democratic Spain. Cambridge, Harvard University Press.

Portes A. e J. Walton (1975), Urban Latin America: The Political Condition from Above and From Below, Austin, University of Texas Press.

Przeworski, A. (1988), Capitalism, Democracy Pacts, Revisited, Chicago, University of Chicago, maggio, manoscritto.

- (1991), Democracy and the Market. Political and Economic Refor$m s$ in Eastern Europe and Latin America, Cambridge, Cambridge University Press.

Rustow, D. (1970), Transitions to Democracy: Towards a Dynamic Model, in «Comparative Politics», II, 3, pp. 337-363.

Sofer, E.F. (1980), Recent Trends in Latin American Historiography, in «Latin American Research Review», n. 1, pp. 167-176.

Spalding, H.A. Jr. (1977), Organized Labor in Latin America: Historical Case Studies of Workers in Dependent Societies, New York, New York University Press.

Tavares de Almeida, M.H., S. Magnani e W. Keller (1985), Sindicalismo brasileiro y pacto social, in Dos Santos (a cura di), pp. 161188.

Tezanos, J.F., R. Cortarelo e A. de Blas (a cura di) (1989), La Transición democrática española, Madrid, Sistema.

Thompson, A. (1995), La partecipazione sindacale nei processi di concertazione sociale in America Latina: analisi di un dibattito, in «Sociologia del Lavoro», n. 55, pp. 17-34.

Tilly, C. (1978), From Mobilization to Revolution, New York, Random House.

Tironi, E. (1988), Sindicalismo y concertación social: alcances teóricos, in PREALC (a cura di), Política económica y actores sociales. La concertación de ingresos de empleo, Santiago, Ilo, pp. 234-257.

Touraine, A. (1988), L'evolution du syndicalisme en Amérique latine, in «Revue Francaise de Sociologie», XXIX, 1, pp. 117-140.

Valente, C.M. (1979), The Political, Economic, and Labor Climate in Venezuela, Philadelphia, University of Philadelphia Press. 
Valenzuela, J.S. (1988), Labor Movements in Transitions to Democracy: a Framework for Analysis, Notre Dame, The Helen Kellogg Institute for International Studies, working paper n. 104.

- (1994) La réforme des relations État-syndicats en Amérique Latine, in C.I. Bradford (a cura di), L'État dans l'Amérique latine, Paris, Ocde, pp. 143-161.

Waisman, C. (1982), Modernization and the Working Class, The Politics of Legitimacy, Austin, University of Texas Press.

Zapata, F. (1993), Autonomía y Subordinación en el Sindicalismo Latinoamericano, Città del Messico, El Colegio de Mexico. 